\title{
Die Kritik am Arzt im Mittelalter - Beispiele und Tendenzen vom 6 . bis zum 12. Jahrhundert
}

von Klaus Bergdolt

\section{ZUSAMMENFASSUNG}

Im mittelalterlichen System der «artes» nahm die Medizin nur den Rang einer «mechanischen» Kunst ein. Diese Degradierung der Heilkunde wurde durch vielfältige Kritik am ärztlichen Beruf gefördert. Christliche Autoren argumentierten, die Medizin schöpfe aus heidnischen Quellen. Später stützten sich die Kritiker gerade auf antike Autoren wie Cato oder Plinius, welche die Notwendigkeit des Arztberufs bestritten hatten. Vielerorts galten Krankheiten als Gottesstrafen, ihre Heilung als Anmassung. Verteidigungsschriften wie das Lorscher Arzneibuch des 8. Jahrhunderts lassen weitere Kritikpunkte der Gegner rekonstruieren. Auch im islamischen Kulturkreis waren die Arzte vielfach Angriffen ausgesetzt. Im 12. Jahrhundert zeigte sich im Westen auch Unzufriedenheit mit dem neuen, an der Universität ausgebildeten Arzttypus, der sich auf die scholastische Tradition berief und in den Augen der Gegner die Praxis vernachlässigte. Dieser intellektuelle «Arzt-Philosoph» wurde auch von konservativeren Kollegen wie Guido von Arezzo angefeindet.

\section{Die Medizin als «ars mechanica»}

In der mittelalterlichen Hierarchie der «artes» nahm die Medizin einen geringen Rang ein. Bereits Martianus Capella hatte in seinem allegorischen Lehrbuch «De nuptiis Philologiae et Mercurii» (um 430), das die freien Künste für lange Zeit «kanonisierte», die Heilkunde übergangen. Zusammen mit handwerklichen Tätigkeiten wie dem «lanificium», der «armatura», «navigatio», «agricultura» und «venatio» erscheint die «medicina» - neben der «theatrica» - an letzter Stelle der «artes mechanicae», die Hugo von St. Victor im 12. Jahrhundert zusammenfasste ${ }^{1}$. Ungeachtet der Tatsache, dass Isidor von Sevilla (560-636) und einige andere ${ }^{2}$ Autoren sie als «secunda philosophia» rühmten, wurde die Kunst der Ärzte von den meisten Intellektuellen des Früh- und Hochmittelalters verachtet. Diese Degradierung war 
freilich nicht nur formal-wissenschaftshistorisch bedingt. Sie wurde über Jahrhunderte durch eine spezifische Tradition der Arztkritik genährt und gefördert.

Obgleich die Krankenpflege seit alters zu den Pflichten christlicher «caritas» gehörte (vielen Klöstern und Gemeinden war so seit dem 4. Jahrhundert ein Hospiz angegliedert ${ }^{3}$ ), steckte die wissenschaftliche Medizin nach dem Untergang des römischen Reichs und dem Sieg des Christentums in einer Krise. Da sie aus antiken, also heidnischen Quellen schöpfte, musste sie sich dem Vorurteil aussetzen, über wenig «Wahrheit» zu verfügen ${ }^{4}$. Es ist immerhin bemerkenswert, dass noch im 14. Jahrhundert einer der Vorwürfe Petrarcas die «averroistische», d. h. heidnisch-gottlose Verstrickung der Heilkunde betraf ${ }^{5}$. Zwar hatten Persönlichkeiten wie Cassiodor (490-580), ein Zeitgenosse Benedikts von Nursia, den Mitbrüdern nicht nur die Pflege schöngeistig-klassischer, sondern ebenso naturwissenschaftlicher und medizinischer Texte empfohlen ${ }^{6}$, doch brachte der - übrigens bereits durch die Kirchenväter legitimierte - Versuch, heidnisch-antikes Bildungsgut in die christliche Theologie zu integrieren ${ }^{7}$, für die Medizin ein neues Problem: Man begeisterte sich in den Klosterbibliotheken so sehr für den klassischen Stil Ciceros, dass die oft in dürftigem Latein verfassten medizinischen Fachtexte einer gewissen Verachtung anheimfielen. Cassiodor, der das System der freien Künste «wie den Baum im Samenkorn» in den biblischen Erzählungen vorgegeben sah $^{8}$, verteidigte zwar Krankenpflege und Hospitalsystem, ja empfahl Mönchsärzten, die kein Griechisch beherrschten, lateinische Übersetzungen medizinischer Klassiker wie Hippokrates, Galen oder Dioskurides, nahm aber die Heilkunde nicht in den auf den «artes liberales» fussenden Lehrplan seines Modellklosters Vivarium auf ${ }^{9}$. Die Tatsache, dass die Medizin - auch von Boethius und Martianus ${ }^{10}$ - nicht den freien Künsten zugeordnet wurde, behinderte zudem lange Zeit die stilistische Emanzipation ihrer Fachprosa. Während man für die «artes liberales» bald eine an der römischen Schriftsprache ausgerichtete wissenschaftliche Fachsprache entwickelte, waren die medizinischen Übersetzungsliteraturen der Langobardenzeit durch «eine am Sprechlatein orientierte vorromanische oder schon romanische vulgäre Sprache» (Gerhard Baader ${ }^{11}$ ) gekennzeichnet. Zweifellos trug dieses ästhetisch-stilistische Manko zur Degradierung der Heilkunde als «ars mechanica» bei. Es war so verständlich, dass im 11. Jahrhundert ärztliche Autoren wie Gariopontus, vor allem aber Alphanus von Salerno $^{12}$ versuchten, die Fachliteratur der tradierten lateinischen Schriftsprache anzugleichen, d.h. für die Medizin das zu erreichen, was Jahrhun- 
derte zuvor «bereits Boethius für die artes und Johannes Scotus Eriugena für die Theologie getan hatten (Baader ${ }^{13}$ ). Diese neue, an der Schriftsprache orientierte medizinische Terminologie musste sich freilich unter unzähligen im Vulgärlatein tradierten Werken behaupten und wurde von den Humanisten des 14. Jahrhunderts bezeichnenderweise übersehen.

\section{Beispiele der Arztkritik im frühen Mittelalter}

Ein weiteres Motiv mittelalterlicher Medizinkritik stellt natürlich die Lektüre antiärztlich eingestellter antiker Autoren wie Plinius, Cato oder Martial dar ${ }^{14}$. Tatsächlich zeigte sich die von den Römern angeprangerte fachliche Insuffizienz der Ärzte während der Pest des Iustinian in erschreckender Weise $^{15}$. Aller Welt wurde - ungeachtet der Seuchenproblematik - der qualitative Niedergang der Medizin im Laufe der Völkerwanderung und nach dem Abriss der antiken Kulturtradition vor Augen geführt. Gregor von Tours (538-593), Verfasser der «Historia Francorum», schreibt über den Starstich:

«Wenn sie [d.h. die Ärzte] das Auge aufhalten und mit ihren spitzen Lanzetten hineinschneiden, so lassen sie [einem] die Qualen des Todes [!] vor Augen treten, ehe sie wieder zum Leben verhelfen. Und wenn nicht alle Vorsichtsmassregeln genau befolgt werden, ist es mit dem Sehen überhaupt vorbei... $\nu^{16}$.

Bei einer solchen Geringschätzung der ärztlichen Kunst tritt fast selbstverständlich die für die folgenden Jahrhunderte wichtigste Konkurrenz auf den Plan: die Hilfe der Heiligen und die Gnade Gottes. «Unser lieber Heiliger (der Bischof von Tours denkt natürlich an den hl. Martin)», fährt Gregor fort, «hat nur ein Stahlinstrument, das ist sein Wille, und eine Salbe, das ist seine Heilkraft». Entsprechend bemerkt sein Zeitgenosse Cassiodor:

«Weil die Medizin, wie man lesen kann, von Gott selbst geschaffen ist, heilt der auch die Kranken, welcher Leben ohne Einschränkung gewährt» ${ }^{17}$.

Krankheit wird als Strafe, als Prüfung wie bei Hiob, aber auch als Zuwendung, ja mit Salomon als Liebesbeweis Gottes gedeutet ${ }^{18}$, als Chance, die der Arzt - auf einer völlig falschen, handwerklich-naturwissenschaftlichen Ebene agierend - vereitelt. Zweifellos duldete die Kirche Ansätze des heidnischen Dämonenglaubens, ja erlaubte entsprechende Heilmethoden durch Kleriker bei psychosomatischen Erkrankungen, wobei die «somatischen» 
Ratschläge der «auctoritates» nicht nur als lästige Konkurrenz, sondern als nichtig und hohl empfunden werden mussten ${ }^{19}$. Nach Keil machten magische Suggestivtherapien «mehr als die Hälfte aller mittelalterlichen Heilversuche aus ${ }^{20}$. Als letzte Ursache der Krankheit galt die Sünde, die mit ihr zusammen bekämpft werden muss (Matt. 9,2). Bevor man Ärzte konsultierte, rief man die Heiligen und Gott selbst um Rettung an. Auch Alkuin, der überragende Gelehrte am Hof Karls des Grossen ${ }^{21}$, erinnert die Ärzte in einem «Carmen» daran, dass Heilung und ärztlicher Erfolg nur möglich seien, «wenn euer Wirken vom Segen Gottes begleitet wird»²2, und Bernhard von Clairvaux (gest. 1153) nimmt, wenn auch in strengerer Form, Petrarcas im «Secretum» akzentuierte Philosophie der Weltverachtung ${ }^{23}$ vorweg:

«Hippokrates und seine Jünger lehren das Leben in dieser Welt erhalten, Christus und seine Jünger lehren es verlieren. Welchen von beiden wollt ihr als Lehrern folgen?»

Der Gegner scholastischer Naturwissenschaft und unerbittliche Zeitkritiker fährt fort:

«Epikur gibt der Lust des Leibes, Hippokrates der Gesundheit des Leibes den Vorzug; mein Lehrer [d.h. Christus] predigt die Verachtung beider Dinge».

Für Bernhard bringen nur «unsere Ärzte», d.h. Gott und die Heiligen, Rettung («Heil, nicht Heilung») ${ }^{24}$. So christlich-demütig diese Haltung sein mag, dürfte sie doch auch durch die fachliche Inkompetenz der zeitgenössischen Ärzte gefördert worden sein. Zudem betrachtet Bernhard den scholastischen Wissenschaftsbetrieb als solchen mit Abscheu: «Flieht aus Babylon», warnt er die Pariser Scholaren, «fliehet und rettet eure Seelen. Eilt gemeinsam alle zu den Stätten der Zuflucht [d.h. den Klöstern]». Wie später Petrarca, favorisiert der Zisterzienser das Ideal der Einsamkeit ${ }^{25}$, das mit der ärztlichen Berufshektik unvereinbar erscheint: «Du wirst mehr in den Wäldern als in den Büchern finden. Hölzer und Steine werden dich mehr lehren als irgendein Meister». Bernhards Ordensbruder Petrus von Celle fügt bezeichnenderweise hinzu: «Dort gibt es kein Disputsgewirr, kein Sophismengewebe». Schon im 12. Jahrhundert verdammt selbst ein «Urscholastiker» wie Abälard die «loquacitas» eines Anselm von Canterbury: «Im Phrasendreschen war er bewundernswert ${ }^{26}$. Noch gab es im 12. Jahrhundert wenig Universitäten, doch solche Vorwürfe an die Scholastik trafen die frühe Universitätsmedizin mit. Tatsächlich reflektiert nach Kristeller selbst die Medizinkritik der frühen Humanisten des 14. Jahrhunderts in vielen Einzelheiten Gedanken aus dem Umkreis Bernhards, demgegenüber Wilhelm von 
St. Thierry klagte, es gäbe Leute, die die Erschaffung des Menschen «nicht von Gott, sondern von der Natur, den Geistern und Sternen herleiten» ${ }^{27}$.

Dass Kritik an der ärztlichen Kunst populär und weit verbreitet war, beweist auch eine süffisante Bemerkung Einhards im 22. Kapitel von «De vita Caroli Magni»:

«Seine [d.h. des Kaisers] Gesundheit war immer ausgezeichnet. Nur in den letzten vier Jahren seines Lebens litt er öfters an Fieberanfällen und hinkte schliesslich sogar auf einem Fuss. Trotzdem folgte er weiter lieber seinem eigenen Gutdünken und nicht dem Rat seiner Leibärzte, die er fast hasste, weil sie ihm vorschrieben, dass er das gewohnte Bratenfleisch aufgeben und dafür gekochtes Fleisch essen sollte» ${ }^{28}$.

Isidor von Sevilla, der, wie erwähnt, der Medizin sogar den Rang einer «secunda philosophia» (des Leibes, im Gegensatz zu der der Seele) zubilligte und das Wort «medicina» von «modus» ableitete, sah die zeitgenössischen Ärzte ebenfalls kritisch und warnte vor jeglichem therapeutischen Übereifer: «So erfahren auch die, welche Arzneien ohne Massen zu sich nehmen, viel Verdruss. Masslosigkeit bringt nämlich nie Gesundheit, sondern nur neue Gefahr» ${ }^{29}$. Der Vorwurf der Masslosigkeit, ja Lächerlichkeit medizinischer Heilverfahren, den Isidor vertrat, wird uns im frühen zehnten Jahrhundert bei Rhases wieder begegnen.

\section{Eine Verteidigungsschrift aus dem 8. Jahrhundert}

Eine interessante Verteidigungsschrift der frühmittelalterlichen Medizin stellt das um 790 in der Reichsabtei Lorsch verfasste «Lorscher Arzneibuch» dar. Es sucht u. a. Angriffe zu parieren, die offensichtlich noch im 8. Jahrhundert von christlicher Seite gegen die Heilkunde vorgebracht wurden. Der Verfasser der von Gundolf Keil und Ulrich Stoll neu edierten Handschrift ${ }^{30}$ dürfte, wie Alkuin und Einhard, dem Intellektuellenzirkel am karolingischen Hof angehört haben, wo Gelehrte wie der langobardische Geschichtsschreiber Paulus Diaconus, der Westgotenbischof Theodulf, der irische Mönch Dungal (der übrigens der Medizin tatsächlich den Rang einer «ars liberalis» zubilligte ${ }^{31}$ ) oder Abt Angilbert einen «interfakultären» Gedankenaustausch pflegten ${ }^{32}$. Er gesteht, durch eine entsprechende Kritik veranlasst worden zu sein, die Tätigkeit der Ärzte zu verteidigen («Cogor respondere»). Den Verächtern der Heilkunde hält er entgegen, dass diese durch die Bibel selbst legitimiert sei : 
«Ich bin genötigt, denen zu erwidern, die sagen, ich hätte dieses Buch unnützerweise geschrieben, indem sie behaupten, darin stehe nur wenig Wahres.[..] Wie taub hörte ich nicht auf ihre Worte, weil ich die Notlage der Hilfsbedürftigen für wichtiger erachtete als den Tadel derer, die gegen mich tobten. Deshalb werde ich ihnen erwidern, nicht mit meinen eigenen Worten, sondern mit denen der Schrift. Ist doch die menschliche Heilkunst durchaus nicht zu verschmähen, da feststeht, dass sie in den göttlichen Büchern nicht unbekannt ist.» (Übersetzung Ohlmeyer/Stoll) ${ }^{33}$.

Der Anonymus räumt den Kritikern zwar ein, dass die (für Ärzte unverzichtbare) Lektüre heidnischer Autoren nicht ungefährlich sei, legitimiert sie aber - abgesehen von dem Argument der Bibel - als Suchen eines Goldklumpens «auf einem Misthaufen» (!) ${ }^{34}$. Auch er gehört zu den wenigen Autoren des Mittelalters, die der Medizin den Rang einer «ars liberalis» zubilligen. Die freien Künste dienen aber nicht mehr, wie es bei Boethius und Isidor, in Anlehnung an den klassischen «Kompromiss» des attischen Redners Isokrates (geb. 436 v. Chr.) ${ }^{35}$, der Fall gewesen war, als Propädeutik zur Philosophie, sondern stellen als «logica» (d.h. Dialektik und Rhetorik) bzw. «physica» (d.h. Arithmetik, Geometrie, Musik, Astronomie, Mechanik und Medizin) zusammen mit der «ethica» (d.h. den vier Kardinaltugenden) diese selbst dar ${ }^{36}$. Von der Apostrophierung als «secunda philosophia» durch Isidor einmal abgesehen, blieb eine solche Hochschätzung der Heilkunst jedoch die Ausnahme, und Hugo von St. Victor degradiert sie, wie erwähnt, im 12. Jahrhundert endgültig als «ars mechanica» ${ }^{37}$. Entscheidend bleibt für den Lorscher Autor aber, dass Krankheiten, Arzneien, Heilungen und Wunder unzählige Male in der Bibel beschrieben wurden, wodurch die Integrierung der Medizin in Gottes Heilsplan bewiesen wird ${ }^{38}$. Zudem sei die Heilkunde eine Gabe des hl. Geistes (1. Kor. 12,9). Wenn Ananias befohlen worden sei, Paulus wieder sehend zu machen (Apg. 9,8ff.), und Jesus selbst den Jüngern aufgetragen habe, Kranke zu heilen (Mk. 16,18, Lk. 9,2), könne niemand deren Nachfolger, die Ärzte, tadeln. Der Verfasser des ältesten deutschen Arzneibuchs zitiert seine Kritiker :

«Da pflegen aber einige zu sagen : \Was haben wir es nötig, von Ärzten geheilt zu werden, da wir unseren Kummer auf den werfen, der, wie feststeht, uns umsorgt? Kann der nicht etwa gewähren, dass wir ohne Heilkunde gesund bleiben, der allein schon durch ein Wort alles zu erneuern vermag? $)^{39}$.

Geschickt widerlegt der Anonymus diese Argumentation, die an Gregor, Cassiodor und Alkuin erinnert ${ }^{40}$, und exkulpiert die Ärzte gerade durch die Tatsache, dass, wie die Gegner ja zugeben, Gott selbst letztendlich der Heiler ist : 
«Wenn jemand, obwohl mit einem Heilmittel versorgt, keineswegs die Gesundheit erlangt, so soll dies seiner Schuld oder seiner [von Gott auferlegten] Prüfung zugeschrieben werden, nicht aber der Unerfahrenheit der Ärzte» ${ }^{41}$.

Nicht die Mediziner können so für den Misserfolg haftbar gemacht werden, «denn obwohl vom Herrn, wie man liest, die Arznei verfügt wird, macht er doch selber gesund $»^{42}$.

Das Sammeln von Heilkräutern, das offensichtlich als heidnischer Volksbrauch gebrandmarkt worden war, sieht der Lorscher Autor durch die Worte Pauli legitimiert: «Wer schwach ist, esse Gemüse» (Röm. 14,2). Auch dem kränkelnden Timotheus habe der Apostel therapeutische Ratschläge erteilt und z. B. gegen Magenbeschwerden Wein empfohlen (1. Tim. 5,23). Ordensinterner Kritik wird entgegengehalten, dass sich auch der hl. Germanus wegen seiner schlechten Gesundheit habe behandeln lassen, ebenso Gregor der Grosse, der vor Schmerzen kaum sprechen konnte, in seinem Kloster aber ständig durch einen Mönchsarzt betreut worden sei ${ }^{43}$. Im Alten Testament sei Gott gleichsam zum Arzt des Volkes geworden, als er beim Auszug aus Ägypten Moses und später Elisa schlechtes Wasser in gesundes verwandeln liess (Ex. 15,23 ff. und Kön. II, 2,21 ff.), ebenso später Christus selbst, der unzählige Male Kranke geheilt habe ${ }^{44}$. Kein geringerer als Lukas sei von Paulus als Arzt angeredet worden (Kol. 4,14), und berühmte Märtyrer wie Kosmas und Damian hätten den Heilberuf ausgeübt ${ }^{45}$. Arzt und Arznei bekommen nach dem Lorscher Anonymus ihre Legitimation durch die Bibel bzw. die heiligen Vorbilder, wobei auf Jesus Sirach verwiesen wird: «Ehre den Arzt in der Notlage, denn ihn hat der Höchste geschaffen» (Sir. 31,8) bzw. «Der Höchste hat aus der Erde eine Arznei geschaffen, und ein kluger Mann wird vor ihr nicht scheuen» (Sir. 38,1) ${ }^{46}$. Auch ein Argument Petrarcas wird hier im 8. Jahrhundert vorweggenommen, freilich nicht in antiärztlichem Sinn : «Soll aber sein [d.h. des Kranken] Bemühen nicht fruchten, so nehme er seine Zuflucht zu den Arzneien der Geduld» ${ }^{47}$. Doch erteilt der Autor seinen Ordensbrüdern auch praktische Ratschläge, wobei er die Lektüre von Hippokrates, Galen, Dioskurides und Caelius Aurelianus empfiehlt und dabei eine Passage der «Institutiones» Cassiodors zitiert ${ }^{48}$. Der Lohn für den Arzt, dessen Beruf durch die christliche Barmherzigkeit erhöht wird, ist im Himmel zu erwarten.

Dieser Gedanke wird auch in den Kosmas und Damian betreffenden Kapiteln der Handschrift fortgesetzt, wo Hippokrates und Galen immerhin mit den berühmten Märtyrern auf eine Stufe gestellt werden ${ }^{49}$. Angesichts der christlichen Medizinkritik scheint dies keinesfalls selbstverständlich 
gewesen zu sein. In etwas vorsichtiger Weise schneidet der Anonymus auch das leidige Thema ärztlicher Besoldung an:

«Dem Arzt gehört sein Lohn, solange jeder leidet. Steht der Kranke auf, kommt kein Beutel mehr. Was du dem Arzt schuldest, gib ihm, solange du krank bist, damit dir nicht erneut Übles zustösst. Noch einmal eilt [sonst] keiner zu dir.» ${ }^{50}$.

Schon hier stand der Vorwurf der Geldgier der Ärzte, aber auch - vice versader schleppenden Bezahlung der Patienten Pate. Interessanterweise liefert der Lorscher Autor des 8. Jahrhunderts konkrete Vorschläge zur medizinischen Kostendämpfung, indem er die therapeutische Wirksamkeit «der gewöhnlichen Wiesenkräuter» (anstelle orientalischer Importdrogen) rühmt $^{51}$. Auch hier scheint Kritik laut geworden zu sein!

\section{Beispiele der Arztkritik im Islam}

Im Schrifttum des Rhases (geb. um 865) werden ebenfalls ärztliche Verhaltensweisen angeprangert. Nach Neuburger zeichnete sich der persische Leibarzt des Kalifen von Bagdad durch eine «nüchterne klinische Richtung» aus, die auch seine Kollegenkritik kennzeichnet ${ }^{52}$. Seine Abhandlung «Über die Umstände, welche die Herzen der Menschen von den achtbaren Ärzten abwenden»;, stellt eine fundierte Stellungnahme zu vielfältiger Kritik an der zeitgenössischen Medizin dar, wobei Rhases z.T. sogar Verständnis dafür bekundet ${ }^{53}$. Er weist scharf die naiven (und nicht selten von den Ärzten selbst kultivierten) Traumvorstellungen der Laien vom Wissen der Ärzte zurück: «Zu den Dingen, welche das Volk zu Gegnern gewissenhafter Ärzte machen und den Betrügern in der medizinischen Praxis Vertrauen erwerben, gehört der Wahn, dass der Arzt alles wissen müsse (und) nichts zu fragen brauche» ${ }^{54}$. Rhases unterstellt, dass die schweigenden Kollegen sich heimlich Informationen bei Freunden und Verwandten verschafften, um den Patienten später zu verblüffen und ihren eigenen Ruf zu fördern ${ }^{55}$. Andererseits verurteilt der Perser vehement die Geschwätzigkeit vieler Ärzte, die im Mittelalter einen wahren Topos der Medizinkritik darstellte:

«Das ist Lug und Trug und wird nur durch Kunstgriffe, durch allerlei künstliche Fragen bewirkt, durch welche man den Sinn des Volkes betört» ${ }^{56}$.

Er kommt im übrigen auf ein weiteres interessantes Phänomen zu sprechen, das - wenn auch zu Unrecht - bei Patienten Misstrauen schürt : 
«Ein anderer Grund zur Geringschätzung des Arztes, auch des scharfsinnigsten und erfahrensten, ist der Umstand, dass viele Krankheiten sich zu wenig von der Gesundheit entfernen, also schwer zu erkennen und zu heilen sind; andere, an sich böse, erscheinen äusserlich unbedeutend. Wenn nun der Laie merkt, dass der Arzt an ihrer Heilung zweifelt, so zieht er die sichere Folgerung, dass der Arzt noch weniger von schweren, umfassenden Krankheiten verstehen und sie heilen werde. Dieser auf Analogie gegründete Schluss ist aber falsch. $\aleph^{57}$

Wenn schon, wie Rhases berichtet, der Angestellte eines Krankenhauses, der ein Geschwür am Finger hatte, die Ärzte «verfluchte und beschimpfte» und Heilung «bei Frauen und beim Pöbel» suchte, lässt sich das dürftige Prestige mancher orientalischer Ärzte im 9. Jahrhundert erahnen ${ }^{58}$. Zugleich tritt die Konkurrenz der Laienheiler, Quacksalber und Scharlatane auf den Plan, die auch im Westen eine wichtige Rolle spielten und das Bild des Arztes verzerrten $^{59}$. Immer wurden auch Ärzte gegeneinander ausgespielt. Der zweite Arzt gab dem Erstbehandelnden das Nachsehen und setzte seinen Kollegen ungerechtfertigt der Kritik aus :

«Manchmal bewirkt der geschicktere Arzt eine Besserung, die aber noch nicht sichtbar ist. Der Kranke wird dann einem anderen Arzt übergeben, der nach kurzer Zeit die Heilung vollbringt, und sie wird diesem zugeschrieben.» ${ }^{60}$

Auch die Herrscher scheinen mit ihren Leibärzten nicht immer zufrieden gewesen zu sein. Rhases, der der Legende nach vom Herrscher der Stadt Chorasan $^{61}$ so sehr geschlagen wurde, dass er erblindete ${ }^{62}(!)$, wirbt deshalb auffallend um Unterstützung durch die Obrigkeit:

«Ich bemerke nun, dass es für einen verständigen Fürsten sehr nützlich ist, seinen Arzt nicht zu beunruhigen, ihn zu erfreuen [!], mit ihm viel zu verkehren, auch auszudrücken, dass er für die Heilung unheilbarer Krankheiten nicht verantwortlich sei und für Irrtum und Missgeschick nicht in die Klemme kommen solle.» ${ }^{63}$

Rhases setzte schon durch sein umfassendes Wissen neue Massstäbe in der Medizin. Unerbittlich geisselt er das gestelzte Verhalten vieler Kollegen. Der gewissenhafte Arzt wird es niemals so weit treiben

«wie jene, welche z. B. sagen : (Wer diesen Urin gelassen hat, schlief gestern bei einer alten Frau oder hat auf der rechten Seite gelegen, und zwar so viele Stunden der Nacht $»{ }^{64}$.

Dem genialen Arzt, der als «zweiter Galen» gefeiert wurde, war - wie später Roger Bacon ${ }^{65}$ - der Autoritätsglaube suspekt :

«Die Heilart, wie sie in den Büchern beschrieben wird, steht weit unter der praktischen Erfahrung.» ${ }^{66}$ 
Auch riet er den Medizinern, den persönlichen Bedürfnissen der Patienten entgegenzukommen, ihre psychische Situation zu berücksichtigen ${ }^{67}$. Der Perser steht für die Solidarität und Ethik der nichtscholastischen Medizin, die eben nicht nur durch Ignoranz und Scharlatanerie gekennzeichnet war, freilich im arabischen Orient wohl auch seriöser und «wahrer» erschien als im Westen. Die Apologetik der wahren Heilkunst und des ehrlichen Arztes lag ihm besonders am Herzen. Der Oberflächlichkeit und Angeberei mancher Kollegen setzt er die Persönlichkeit des moralisch integren, bescheidenen und erfahrenen Mediziners entgegen, der freilich nicht häufig zu finden ist. Kein Wunder, dass, wie Rhases kritisch bemerkt, der Patient am schlechtesten wegkommt, der möglichst viele Ärzte konsultiert ${ }^{68}$.

Empfehlungen an Patienten und Kollegen, die kritischen Stimmen vorbeugen sollten, gab Ende des 10. Jahrhunderts auch der in Ägypten und Kairouan tätige Arzt Isaak Iudaeus in einer (ihm zugeschriebenen) Abhandlung «Führung der Ärzte» ${ }^{69}$ :

«So du einen Arzt über jede Krankheit, über die du ihn befrägst, sofort Auskunft erteilen und seiner Heilmethode sich noch rühmen siehst, so halte ihn für einen Toren.[...] Ebensowenig wie der Arzt sich in seinem Vorgehen übereilen soll, darf er lässig und saumselig sein, da die meisten Kranken ihm dazu keine Zeit lassen.»

Kritik klingt auch in folgendem Satz an:

«Es gehört zum Charakter des [guten] Arztes, dass er [...] kein Schlemmer und Prasser werde» ${ }^{70}$.

Die eigene Erkrankung disqualifiziert den Arzt (!):

«Auch ist es beschämend für ihn, an einer langwierigen Krankheit zu laborieren, da sonst

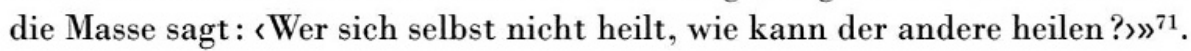

An das Lorscher Arzneibuch erinnert folgende Anweisung:

«Dein Honorar von dem Kranken bestimme dann, wenn seine Krankheit im Zunehmen begriffen und am heftigsten ist. Denn sobald er geheilt ist, vergisst er, was du an ihm geleistet hast»,

und:

«Je mehr du für deine Behandlung fordern, je teurer du deine Kuren ansetzen wirst, desto höher werden sie in den Augen der Leute steigen .... $>^{72}$.

Man bekämpfte die Kritik, man provozierte sie aber auch. 


\section{Kritische Stimmen im 12. Jahrhundert}

Denken wir an die von Henri d'Andeli im 12. Jahrhundert angeprangerten «artes lucrativae», denen auch die Heilkunde zugerechnet wurde ${ }^{73}$, erscheinen solch zynische Empfehlungen auch im Westen populär gewesen zu sein:

«Tactus avaritia), von Habsucht ergriffen, geht der Arzt allem anderen als christlicher Mildtätigkeit nach, und weit davon entfernt, sich karitativen Grundsätzen zu unterwerfen, sieht er im Kranken keineswegs (immer) einen 'pauper Christus', dem es selbstlos zu helfen gilt» $\left(K_{\text {Keil }}^{74}\right)$.

In seinem «Metalogicon» nimmt der zeitgleiche Johannes von Salisbury (1110-1180) die Mediziner aufs Korn ${ }^{75}$.

«Andere nämlich, die ihre mangelnde Begabung für die Philosophie erkannten, gingen nach Salerno oder Montpellier, erlernten dort die Medizin und kehrten plötzlich als ebenso gute Ärzte zurück, wie sie zuvor Philosophen waren»,

bemerkt der Schüler des Bernardus Silvestris spöttisch und nimmt gleich einen Hauptvorwurf Petrarcas gegenüber den Medizinern vorweg:

«Sie geben mit Hippokrates und Galen an, werfen mit früher niemals gehörten Vokabeln um sich, kommentieren alles mit ihren 'Aphorismen' und machen den Menschen die Köpfe mit dem Donnergetöse ihrer Worte heiss» ${ }^{76}$.

Göttergleich bestimmen sie,

«wann Krankheit vorliegt oder Gesundheit oder ein Zwischenstadium. [...] Wenn man sie hört, glaubt man, sie könnten Tote erwecken, denn sie halten sich Äskulap und Merkur ebenbürtig..1 ${ }^{77}$

Sarkastisch fährt Johannes fort:

«In meiner Bewunderung für soviel Geist werde ich freilich unsicher und verwirrt, wenn sie sich untereinander lautstark ihre Meinungen vorhalten und herumstreiten. [Denn] eines weiss ich: Gegenteilige Auffassungen können nicht wahr sein» ${ }^{78}$.

Der ätzende Kritiker der Scholastik klagt auch die «Praktiker» an, die er bezeichnenderweise von den Arzt-Philosophen trennt ${ }^{79}$. Er wage es freilich nicht, sie zu kritisieren :

«Ich hüte mich, etwas Schlechtes über sie zu sagen. Zu häufig bin ich nämlich ihr Patient» ${ }^{80}$.

Auch für Johannes ist der Arzt nur Vollstrecker. Trotz aller Kritik erinnert er an den (auch vom Lorscher Anonymus zitierten) Ausspruch des Ecclesiasticus, «dass der weise Mann die Heilkunde nicht verachten darf, weil sie von Gott selbst kommt» ${ }^{81}$. Freilich wird die sprichwörtliche Gewinnsucht der 
Ärzte gegeisselt, deren alte «Berufsweisheit» Johannes ironisch kolportiert: «Dum dolet, accipe...» ${ }^{82}$. Zuweilen ist seine Kritik auch bitterer:

«Wenn jemand das Pech hat, in die Hände [der Ärzte] zu fallen, führen sie Gründe an, die es notwendig erscheinen lassen, dass sein Leben nicht verlängert werden darf» ${ }^{83}$.

Auch die therapeutische Effizienz wird in Frage gestellt:

«Man sagt, dass sie einem, den sie durch langes Fasten gequält haben, vor dem Tod Suppen und ebenso nutzlose wie wohlschmeckende Speisen verabreichen ${ }^{84}$.

Vielsagend, und scheinbar nicht ohne Angst, bemerkt Johannes:

«Du erwartest vielleicht, dass ich erzähle, was das Volk dazu meint, dass es Leute gibt, die Menschen berufsmässig töten. [...] Ich werde mich hüten, diese Verbrechen überhaupt zu erwähnen» ${ }^{85}$.

\section{Die Bedenken eines Praktikers}

Vor allem im 12. Jahrhundert akzentuierte sich die mittelalterliche Arztkritik, als die traditionelle Medizinerausbildung, welche sich seit Cassiodor auf die «artes» stützte, ins Wanken geriet. Während z. B. Isidor im 7. Jahrhundert die Beherrschung der «artes liberales» als zentralen Bestandteil ärztlicher Kunst definiert hatte, dienten die freien Künste an den Universitäten nur als Propädeutik-Stoff. Im Mittelpunkt der medizinischen Ausbildung standen jetzt die arabischen bzw. durch die Araber tradierten antiken Autoritäten. Um 1180 äussert Guido von Arezzo der Jüngere ${ }^{86}$, ein Schüler des Chirurgen Roger Frugardi aus Parma, massive Kritik an dieser Entwicklung $^{87}$. Guidos Argumente leiten einen Methodenstreit ein, der nach Gründung der medizinischen Fakultäten zwischen den Anhängern der herkömmlichen Ausbildung und den scholastischen, d.h. universitätsgebildeten, durch die Araber beeinflussten, philosophisch beschlagenen Ärzten unausweichlich schien. Der Disput reflektierte den Paradigmenwechsel (Kuhn ${ }^{88}$, Schipperges $^{89}$ ) des europäischen Bildungssystems: Die Verfechter des früh- und hochmittelalterlichen «artes»-Systems waren nicht bereit, sich kampflos dem neuen Bildungsideal unterzuordnen, welches die freien Künste zu einführenden Fächern degradierte. Im «Liber mitis» geisselt Guido ebenso den begeisterten Arabismus seiner Kollegen wie ihre kritiklose Akzeptanz

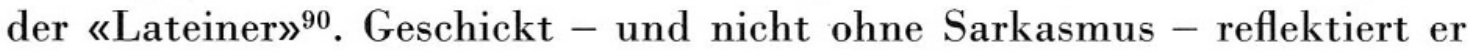
deren Einstellung: 
«Sehen sie [d.h. die scholastischen Ärzte] einen Mann, der nicht den trockenen Johannitius erlernt, der nicht in Montpellier, von alters her erbärmlich, und im wahnsinnigen Salerno studiert hat, so zeigen sie Verachtung in Gesicht und Rede.»11

Guido scheut sich allerdings auch nicht, das Zerrbild, welches die Öffentlichkeit bis in die Zeit Petrarcas vom Arzt hatte, zu plakatieren:

«Sie beschauen ihre jämmerlichen Urinproben (miserabiles micturas) auf öffentlichen Plätzen und schütteln die stinkenden Harngläser, damit andere Narren mit ihren Urinen zu ihnen hinlaufen ${ }^{92}$.

Er unterstellt den Kollegen, mit ihren roten Hüten «Vogeltäuscher» zu sein und dem «Gift des Neides» zu erliegen ${ }^{93}$. Unter Berufung auf Johannitius nimmt er ein Argument des Isaak auf:

«Wirst du [am Krankenbett] gefragt und antwortest sofort, so gerätst du in Verdacht, denn der Rede des Gebildeten geht die Abwägung voraus» ${ }^{94}$.

Entscheidend aber ist wiederum der Vorwurf der dümmlichen Autoritätsgläubigkeit, den bereits Rhases erhoben hatte, der aber besonders von Petrarca später den scholastischen Medizinern vorgehalten wird:

«[Meine Rede] mag die Irrlehren der Mehrzahl der Ärzte des Altertums zuschanden machen und all die vielen Lügen ausrotten, die sich in der Heilkunde nicht aus Kenntnis der Kunst, sondern durch falschen Gebrauch der lateinischen Autoritäten eingenistet haben» ${ }^{95}$.

Ebenso bezichtigt Guido die Ärzte der Arroganz: «Faktisch wissen sie nichts, ja sie rühmen sich (nur) ihres hohlen Wissens» ${ }^{96}$. Er tadelt auch deren sprichwörtliche Logorrhoe, die immer wieder Gegenstand allgemeinen Spotts war : «Sie reden nur, doch mit dem Abführen wird's nichts» ${ }^{97}$. Interessant erscheint die Verachtung der «artes» durch die Scholastiker, die der Arzt aus Parma seinen Kollegen vorwirft, so etwa der «geometria»:

«Sie sagen, was nützt es einem Arzt, ein Quadrat in ein Fünfeck zu verwandeln oder gar mit einem Zirkel Umfang und Radius eines Kreises herauszufinden ?» ${ }^{98}$

Angesichts der Tatsache, dass die freien Künste als propädeutische Disziplinen an den Universitäten eine durchaus wichtige Rolle spielten und späterzumindest in Italien - von Medizinprofessoren gelehrt wurden $(!)^{99}$, erscheint die geschilderte Haltung der Scholastiker erstaunlich und wohl auch überzeichnet. Auch war Guido selbst von der Scholastik selbst keinesfalls unbeeinflusst. Trotz seiner Warnung vor den «auctoritates» griff auch er auf tradierte Schriften zurück, wobei er sich offensichtlich auf durch Gerhard von Cremona aus dem Arabischen übersetzte Werke stützte, die er in Italien 
wahrscheinlich als erster, d.h. noch vor den Salernitaner Kathederärzten rezipierte ${ }^{100}$. Im Banne des damals in Salerno wohl noch unbekannten Avicenna fühlte er sich dem Universitätsbetrieb freilich überlegen ${ }^{101}$. Von der scholastischen Medizin trennten den Aretiner so weniger die Autoritäten $^{102}$ als die Ablehnung der Hochschulen. Tatsächlich waren die Übergänge im 12. Jahrhundert recht fliessend, d.h. weder war die «vorscholastische» Medizin uneingeschränkt mit den «artes» verbunden noch die «scholastische» absolut gegen diese eingestellt. Petrarca hätte - und dies scheint sehr bezeichnend - später das oben angeführte «scholastische» Argument auch gegen die Scholastik verwenden können ${ }^{103}$.

Angesichts der Tatsache, dass sich die scholastische Medizin in den folgenden Jahrhunderten in Europa etablieren konnte, ist Guido als konservativer, letztlich gescheiterter Kritiker des ärztlichen «Zeitgeistes» anzusehen. Da er in vielem aber auch Kritikpunkte Petrarcas vorwegnahm und seine streng antischolastische Haltung sich bei den Humanisten am Ende durchsetzte, war sein Arztbild zugleich extrem fortschrittlich und - seit dem 14. Jahrhundert - für die Zukunft auch richtungsweisend.

Mit weiteren Universitätsgründungen im 13. Jahrhundert bzw. der Etablierung neuer medizinischer Fakultäten wird die Kritik am Arzt in der Folgezeit immer mehr zur Kritik am akademisch gebildeten Theoretiker, am Arzt-Philosophen, der die Heilkunde in Europa nun mehr entscheidend prägte. Die Universitätsbildung der Ärzte wird schliesslich durch die Medizinalordnung Friedrichs II. (1231) sanktioniert. Ein langer Findungsprozess ging damit zu Ende, der in seinen verschiedenen Stadien von Ärzten und Laien heftig kritisiert worden war. 


\section{Anmerkungen}

1 Vgl. zu Martianus Capella Ernst Robert Curtius, Europäische Literatur und lateinisches Mittelalter. Bern und München 1967, S.47-49; ferner Heinrich Schipperges, Der Garten der Gesundheit. Medizin im Mittelalter. München und Zürich 1985, S. 170-185. Zu Hugo von St. Victor vgl. Ch. H. Buttimer, Hugonis de Sancto Victore Didascalicon. Washington 1939, Bd. II, S. 20-25; ferner Paul Oskar Kristeller, Das moderne System der Künste, in: Humanismus und Renaissance II: Philosophie, Bildung und Kunst (= Uni-Taschenbücher 914). Hg. von Eckhard Kessler, übersetzt von Renate Schweyen-Ott. München 1974, S. 173 .

2 Vgl. Isidori Hispalensis Episcopi etymologiarum sive originum libri XX (liber IV, 13,1). Ed. W. M. Lindsay. Oxford 1966, ohne Seitenangabe: «Quaeritur a quibus quare inter ceteras liberales disciplinas Medicinae ars non contineatur. Propterea quia illae singulares continent causas, ista vero omnium. [...] Hinc est quod Medicina secunda philosophia dicitur». Zu Vinzenz von Beauvais, der ebenfalls von der «secunda philosophia» spricht, vgl. Schipperges (1985) (wie Anm. 1), S. 187.

3 Die Konzilien von Nikäa (325) und Karthago (398) forderten die christlichen Kommunen auf, Hospize für Kranke, Arme und Alte zu errichten, vgl. Gundolf Keil/Adelheid Platte, Zur Heilkunde im frühen Mittelalter, in: Das Lorscher Arzneibuch. Klostermedizin in der Karolingerzeit. Ausgewählte Texte und Beiträge. Lorsch 1989, S. 15.

4 Vgl. Albert Ohlmeyer, Die Rechtfertigung der Heilkunde, in: Das Lorscher Arzneibuch (1989) (wie Anm. 3), S. 48.

5 Vgl. etwa - unter vielen ähnlichen Aussagen des Dichters - den Altersbrief Nr.6/15, vgl. Lettere Senili di Francesco Petrarca. Hg. von Giuseppe Fracassetti. Florenz 1892, Bd. II, S. 425: «Quel rabbioso cane ch' è Averroe, il quale agitato da infernale furore, con empi latrali, e con bestemmie da ogni parte raccolte, oltraggia e lacera il santo nome di Cristo e la cattolica fede.»

6 Vgl. R.A.B. Mynors (Ed.), Cassiodori Senatoris Institutiones. Oxford 1937, S. 78 f.

7 Vgl. August Buck, Die humanistische Tradition in der Romania. Bad Homburg, Berlin, Zürich 1968, S.40.

8 Vgl. Curtius (1967) (wie Anm. 1), S.51. Cassiodors «Institutiones» stellten das erste christliche Handbuch des kirchlichen Wissens und der weltlichen «artes» dar. Der Abt von Vivarium konstatiert, dass deren Keim «von Urbeginn her in der göttlichen Weisheit und in der heiligen Schrift angelegt ist» (Curtius).

9 Vgl. Hanno Caprez, Krankenpflege und Heilkunde der Mönche bis zum 9. Jahrhundert, in: Ciba-Zeitschrift (Basel) XI, 126 (1951), S.4651 f.

10 Die aktuellste Zusammenfassung von Boethius' System der «artes liberales» stammt von Margret Gibson, vgl. Boethius, His Life, Thought and Influence. Hg. von Margret Gibson. Oxford 1981, S. 73-274 (Beiträge von Jonathan Barnes, Osmund Lewry, John Caldwell, David Pingree, Alison White, John Mair und Margret Gibson). Zu Martianus Capella vgl. Schipperges (1985) (wie Anm. 1); ferner Curtius (1967) (wie Anm. 1), S. 47 f.

11 Vgl. Gerhard Baader, Die Entwicklung der medizinischen Fachsprache im hohen und späten Mittelalter, in : Fachprosaforschung. Acht Vorträge zur mittelalterlichen Artesliteratur. Hg. von Gundolf Keil und Peter Assion. Berlin 1974, S. 94.

12 Baader, loc. cit. S. $97 \mathrm{f}$. 
13 Vgl. Baader, loc. cit. S. 98.

14 Vgl. etwa Plinius, Naturalis historia XXIX, 8. Wohl durch die Lektüre des Plinius wurde Petrarca auch von Cato beeinflusst. Zu Martial vgl. Egbert Farber, Mittelalterliche Kritik am Arzte. Inaug. Diss. Bonn 1950, S. 5 f.

15 Zur Pest des Iustinian vgl. Karl-Heinz Leven, Die «Iustinianische Pest», in: Jahrbuch des Instituts für Geschichte der Medizin der Robert-Bosch-Stiftung 6 (1987), S. 137-161.

16 Übersetzung nach Keil/Platte (1989) (wie Anm. 3), S.17; vgl. auch Farber (1950) (wie Anm. 14), S. 16.

17 Vgl. Mynors (1937) (wie Anm. 6), S.78: «Nam quamvis medicina legatur a Domino constituta, ipse tamen sanos efficit, qui vitam sine dubitatione concedit.»

18 Vgl. Ohlmeyer (1989) (wie Anm. 4), S. 53 (zu Hiob) und S. 54 (zum Prediger Salomon, $3,12)$.

19 Vgl. hierzu Paul Diepgen, Über den Einfluss der autoritativen Theologie auf die Medizin des Mittelalters, in: Akademie der Wissenschaften und der Literatur. Abhandlungen der geistes- und sozialwissenschaftlichen Klasse. Wiesbaden 1958, S.3-20, besonders S. 5-9.

20 Vgl. Keil/Platte (1989) (wie Anm. 3), S. 17.

21 Zur Sünde als Krankheitsursache vgl. Diepgen (1958) (wie Anm. 19), S. 8; zu Alkuin vgl. Dieter Schaller, in: Verfasserlexikon zur deutschen Literatur des Mittelalters, 2. Aufl. Berlin und New York. Bd.I, 1978, Sp.241-253; ferner Curtius (1967) (wie Anm. 1), S. 57 f.; ferner Eduard Norden, Die antike Kunstprosa. Vom VI. Jahrhundert v. Chr. bis in die Zeit der Renaissance. 5. Aufl. 1958, Bd.II, S.697.

22 Vgl. Alkuin, Carmina, zit. nach Farber (1950) (wie Anm. 14), S.19: «...Et tamen, o medici, cunctis impendite gratis, ut manibus vestris adsit benedictio Christi.»

23 Vgl. Francesco Petrarca, De secreto conflictu curarum mearum (= Opere latine di Francesco Petrarca, hg. von Antonietta Bufano, 1). Turin 1975, S.60-82.

24 Vgl. hierzu Katharina Wäckerlin-Swiagenin, Bernhard von Clairvaux und sein Verhältnis zur Wissenschaft, in: Sudhoffs Archiv 60 (1976), S. 13-32, hier zit. S.26.

25 Vgl. etwa die Verteidigung der «vita solitaria» im vierten Buch der «Invectiva contra medicum», ferner die Abhandlungen «De rebus memorandis» (nach 1343) und «De otio religiosorum» (1347). Das Zitat findet sich bei Jacques Le Goff, Die Intellektuellen im Mittelalter. Aus dem Französischen übersetzt von Christiane Kayser. Stuttgart 1987, S. 29.

26 Vgl. Le Goff, loc. cit. S. 29 (Bernhard, Petrus von Celle) u. 43 (Abälard).

27 Vgl. Paul Oskar Kristeller, Petrarcas Stellung in der Geschichte der Gelehrsamkeit, in: Italien und die Romania in Humanismus und Renaissance. Festschrift für Erich Loos. Hg. von Klaus Hempfer und Enrico Straub. Wiesbaden 1983, S.119; ferner zu Wilhelm von St. Thierry Le Goff, loc. cit. S. 54 .

28 Vgl. Einhard, Vita Caroli Magni, Das Leben Karls des Grossen 22. Lateinisch-Deutsch von Evelyn Scherabon-Firchow. Stuttgart 1981 (= Reclams Universalbibliothek 1996). Aus dieser Anekdote lässt sich allerdings nicht schliessen, Karl sei der Medizin feindlich gegenübergestanden, vgl. Keil (1989) (wie Anm. 3), S. 20. Jedenfalls förderte der Kaiser die ärztliche Ausbildung im Capitulare von Thionville («De medicinali arte, ut infantes hanc discere mittantur»), vgl. Max Neuburger, Geschichte der Medizin. Stuttgart 1911, Bd.II, S.267.

29 Vgl. Lindsay (1966) (wie Anm. 2): «Inmoderatio enim omnis non salutem, sed periculum affert.» 
30 Vgl. Das Lorscher Arzneibuch. Faksimile der Handschrift Msc. Med. 1 der Staatsbibliothek Bamberg. Hg. von Gundolf Keil. Stuttgart 1989.

31 Vgl. Caprez (1951) (wie Anm. 9), S. 4653.

32 Zu Angilbert vgl. Dieter Schaller, in: Verfasserlexikon (1978) (wie Anm. 21), Bd.I, Sp. 358-363.

33 Vgl. Ohlmeyer (1989) (wie Anm. 4), S.48-50.

34 Ohlmeyer, loc. cit. S. 50.

35 Zu Isokrates vgl. Der kleine Pauly, Lexikon der Antike in fünf Bänden. München 1979, Bd. II, Sp. 1467-1472.

36 Vgl. Das Lorscher Arzneibuch (1989) (wie Anm. 30). Übersetzungsband S. 17 f.

37 Zu Isidor von Sevilla vgl. Anm. 2, zu Hugo von St. Victor Anm. 1. Allerdings setzt Hugos Zeitgenosse Honorius von Autun die Medizin ebenfalls den «artes liberales» gleich, indem er die traditionellen freien Künste um die Medizin, Mechanik und Ökonomik erweitert, vgl. Heinrich Schipperges, Honorius und die Naturkunde des 12. Jahrhunderts, in: Sudhoffs Archiv 42 (1958), S. 71-82.

38 Vgl. Das Lorscher Arzneibuch (1989) (wie Anm. 30), S.17-21 (Übersetzungsband).

39 Lorscher Arzneibuch, loc. cit. S. 19.

40 Zu Gregor vgl. Anm. 16 und oben S. 3; zu Cassiodor Mynors (1937) (wie Anm. 6), S. 78: «Omne quod facitis in verbo aut opere, in nomine Domini Iesu facite, gratias agentes Deo et Patri per ipsum.»

41 Vgl. Das Lorscher Arzneibuch (1989) (wie Anm. 30), Übersetzungsband S.23.

42 Loc.cit. S. 24.

43 Loc. cit. S. 20.

44 Loc. cit. S. $22 \mathrm{f}$.

45 So enthält das Lorscher Arzneibuch einen Abschnitt, in welchem Kosmas, Damian und Galen als gleichbedeutende Autoritäten gefeiert werden, vgl. Das Lorscher Arzneibuch (1989) (wie Anm. 30), Übersetzungsband S.25.

46 Zit. nach dem Lorscher Arzneibuch, loc. cit. S. 23.

47 Vgl. loc.cit. S.23f.; auch Petrarca beschwört die Geduld als Heilerin, vgl. Giovanni Petrarca, De remediis utriusque fortunae. Heidelberg 1490, Fol. LXXXIr: «Patientia in adversis efficacissimum atque optimum et saepe unicum remedii genus est.»

48 Vgl. Cassiodor, Institutiones, Mynors (1937) (wie Anm. 6), S. 78 f.: «Quod si vobis non fuerit Graecarum litterarum nota facundia, in primis habetis Herbarium Dioscuridis, qui herbas agrorum mirabili proprietate disseruit atque depinxit. Post haec legite Hippocratem atque Galienum Latina lingua conversos, id est Therapeutica Galeni ad philosophum Glauconem destinata, et anonymum quendam, qui ex diversis auctoribus probatur esse collectus, deinde Caeli Aureli de Medicina et Hippocratis De Herbis et Curis diversosque alios medendi arte compositos, quos vobis in bibliothecae nostrae sinibus reconditos Deo auxiliante dereliqui.»

49 Vgl. Das Lorscher Arzneibuch (1989) (wie Anm. 30), S. 25.

50 Zit. nach dem Lorscher Arzneibuch, loc. cit. S. 25.

51 Vgl. Lorscher Arzneibuch, loc. cit.: «Ist einer reich, möge es eine rechte Gelegenheit zum Gewinn sein, ist er arm, lass dich mit einer Winzigkeit abfinden. Was immer Arabien, was der Indus an Wohlgerüchen darbietet, was immer die Woge des Ozeans herbeiführt, Zimt, Myrrhe, Nardenblatt und prangende Röhrenkassie, Balsam, Weihrauch, Zuckerrohr und 
koryzischen Safran: diese können Spezereienhandlungen grossen Stils sich leisten und ein Haus, das an ungeheurem Reichtum überfliesst. Wir dagegen erfreuen uns an den gewöhnlichen Kräutern der Wiesen, welche das flache Land und die hohen Berge hervorbringen. Seid deshalb gegrüsst, ihr heiligen Berge und Felder der Heimat.»

52 Vgl. Neuburger (1911) (wie Anm. 28), II, S. 168-174. Zu Rhases vgl. auch Biographisches Lexikon der hervorragenden Ärzte aller Zeiten und Völker. 3. Aufl. (1962), Bd. I, S. 170172. Vgl. auch Farber (1950) (wie Anm. 14), S.6-14.

53 Vgl. Neuburger, loc. cit. S. 173; ferner Farber, loc. cit. S. 7.

54 Zit. nach Neuburger, loc. cit. S. 173.

55 Neuburger, loc. cit.

56 Neuburger, loc. cit.

57 Der Text lehnt sich eng an die Übersetzung von Neuburger an, vgl. loc. cit.; ferner Farber (1950) (wie Anm. 14), S. 10.

58 Vgl. Neuburger S.173f.

59 Vgl. hierzu Heinrich Schipperges, Der Scharlatan im arabischen und lateinischen Mittelalter, in: Zur Geschichte der Pharmazie. Geschichtsbeilage der Deutschen Apotheker-Zeitung 12 (1960), S.9-13; Schipperges fasst auch die Vorwürfe des Rhases zusammen.

60 Vgl. Neuburger (1911) (wie Anm. 28), II, S.174; ferner Farber (1950) (wie Anm. 14), S. $11 \mathrm{f}$.

61 Vgl. Farber, loc. cit. S. 6; ferner Neuburger, loc. cit. S. 168.

62 Leider ist die Autobiographie des Rhases verlorengegangen. Rhases war der Überlieferung nach nicht in der Lage, Al-Mansur Experimente vorzuführen, die er in seiner Schrift «Confirmatio artis chimiae» erläutert hatte, vgl. Neuburger, loc. cit.

63 Zit. nach Neuburger S. 175.

64 Neuburger, loc. cit. S. 173.

65 Vgl. Klaus Bergdolt, Der Sehvorgang als theologisches Analogon. Augenanatomie und -physiologie bei Roger Bacon, in: Sudhoffs Archiv (im Druck).

66 Vgl. Neuburger (1911) (wie Anm. 28), II, S. 172. Dennoch fordert Rhases seine Kollegen auf, die Werke der Vorgänger gründlich zu studieren, denn «per mille annos mille scriptores professionem hanc auxerint».

67 Vgl. hierzu die Schrift «Quod medicus non solum prudens esse debeat sed aegrotum desideriis indulgens», ferner die «Aphorismen», wo Rhases den Kollegen nahelegt, auch dann dem Patienten noch Trost zu spenden, wenn die Zeichen des Todes sichtbar werden, vgl. Neuburger, loc.cit. S.172 f., dort Anm. 1.

68 Vgl. loc. cit. : «Qui quamplures medicorum interrogaverit, in errorem incidit plurimum.»

69 Zu Isaak vgl. Neuburger (1911) (wie Anm. 28), II, S.176-178; ferner Biographisches Lexikon (1962) (wie Anm. 52), Bd. III, S. $377 \mathrm{f}$.

70 Vgl. Neuburger, loc. cit. S. 177.

71 Neuburger, loc. cit.

72 Vgl. loc. cit.; ferner oben S. 5-7 (zum Lorscher Anonymus).

73 Vgl. Buck (1968) (wie Anm. 7), S. 82; vgl. auch Albert Gier, Henri d'Andeli, in: Lexikon des Mittelalters IV (1988), Spalte 2135.

74 Vgl. Gundolf Keil, Der Arzt - ein Lügner? Zur Sozialgeschichte mittelalterlicher Standeskritik. Vortrag, gehalten vor dem Fachbereich Medizin der Universität Marburg am 2.12.1971 (Ms. im Institut für Geschichte der Medizin der Universität Würzburg). 
75 Zu Johannes v. Salisbury vgl. Buck (1968) (wie Anm. 7), S.44; ferner Norden (1958) (wie Anm. 21), S. 712-717; ferner Neuburger (1911) (wie Anm. 28), II, S. 324 f.

76 Vgl. Neuburger, loc.cit. S.324: «Alii autem, suum in philosophia intuentes defectum, Salernum vel ad Montem Pessulanum profecti, facti sunt clientuli medicorum, et repente quales fuerant philosophi, tales in momento medici erupuerunt», ferner: «Hippocratem ostentant, aut Galenum, verba proferunt inaudita, ad omnia suos loquuntur Aphorismos, et mentes humanas velut afflatas tonitruis [...] percellunt.»

77 Vgl. loc. cit.: «... sanitatis, aegritudinis et neutralitatis censores sunt... Cum eos audio, videntur mihi posse mortuos suscitare nec Aesculapio Mercuriove creduntur inferiores.»

78 Vgl. loc. cit.: «Verum tamen in ea magna mentis admiratione distrahor et perturbor, quod a se ipsis tanto verborum conflictu et collisione rationum dissiliunt et discordant. Unum profecto scio: Contraria simul vera esse non posse...»

79 D.h. der scholastische «physicus», der sich im 13. Jahrhundert endgültig in der Medizin durchsetzt, war schon im 12. Jahrhundert sehr verbreitet. Ihm stellt Johannes offensichtlich den nichtakademischen Wundarzt gegenüber.

80 Vgl. Neuburger (1911) (wie Anm. 28), II, S.324: «Absit ut de his quicquam perversum dicam. In manus eorum enim ... nimis frequenter incido.»

81 Vgl. Neuburger, loc. cit.

82 Neuburger, loc. cit. S.325.

83 Vgl. loc. cit.: «Sed cum inter manus eorum quis in fata collapsus est, tunc necessarius producent rationes, quibus appatebit quod vita eius non fuerat ulterius protendenda.»

84 Vgl. Neuburger, loc. cit.: «Et ut dicitur, quos longa afflixerunt inedia, iam mortius sorbitiunculas faciunt et inutiles et delicatos praeparant cibos.»

85 Vgl. loc. cit.: «Expectas forte, ut dicam, quod dicit populus, quia hi sunt, qui homines officiosissime occidunt [...] Absit [...], ut hanc contumeliam proferam.»

86 Im Gegensatz zu dem älteren Musiker gleichen Namens, dem Erfinder der Notenschrift.

87 Zu Guido d'Arezzo vgl. Konrad Goehl, Guido d'Arezzo der Jüngere und sein «Liber mitis» ( = Würzburger Medizinhistorische Forschungen 32). Pattensen/Hann. 1984; ferner Gundolf Keil und Konrad Goehl, Guido d'Arezzo der Jüngere und die «Disputà delle arti», in : Atti del XXXII congresso nazionale della Società Italiana di Storia della medicina, hg. von Loris Premuda. Padua 1987, S.97-110; ferner Gundolf Keil, Guido d'Arezzo der Jüngere und die Medizinschulen seiner Zeit, in: Verhandlungen des XXX. Internationalen Kongresses für Geschichte der Medizin. Hg. von Hans Schadewaldt. Düsseldorf 1988; zur Kritik Guidos vgl. Goehl, loc. cit., I, S. 12. Zu Roger Frugardi vgl. Adalberto Pazzini, Ruggero di Giovanni Frugardo, maestro di chirurgia a Parma e l'opera sua. Rom 1966 (= Collana di Pagine di storia della medicina 13).

88 Zum Begriff des «Paradigmenwechsels» vgl. Thomas S. Kuhn, Die Entstehung des Neuen, Studien zur Struktur der Wirtschaftsgeschichte. Hg. von Lorenz Krüger. Übersetzt von Hermann Vetter. Frankfurt/M. 1977, S. 389-420.

89 Bezüglich der Medizingeschichte spricht Schipperges am Beispiel der Krankheitskonzepte Galens, Paracelsus' und Virchows ebenfalls von «Paradigmata», vgl. Heinrich Schipperges, Homo patiens. Zur Geschichte des kranken Menschen. München/Zürich 1985, S. 101-179 («Die tragenden Paradigmata»).

90 Vgl. Goehl (1984) (wie Anm. 87), I, S.22 f.

91 Vgl. loc. cit. S. 231: «Si quem nisi in arido Johannitio vel ab antiquis miserabili Pessulano 
et vesano Salerno studentem vident, despiciunt ore nec corde tamen [...]» (Übersetzung Goehl).

92 Vgl. loc. cit.: «Aspiciunt miserabiles mincturas in plateis, concutiendo foetida urinalia, ut alios fatuos ad se currere faciant cum urinis.»

93 Vgl. Goehl, loc. cit. S. 232.

94 Vgl. Goehl, loc.cit.: «Si interrogatus velociter respondeas, suspectus es, quia sermonem eruditi praecessit deliberatio.»

95 Vgl. Goehl, loc.cit. S.231: «[oratio,] quae confundat errores plurimum antiquorum medicorum et exstirpet multa mendacia medicinae non ex arte sed per abusum Latinorum introducta.»

96 Vgl. loc. cit.: «[...] qui iure cum nihil sciant de nihilo gloriantur.»

97 Vgl. loc.cit.: «[...] dicunt enim, et non solvunt.»

98 Vgl. loc.cit.: «[...] et dicunt: quid prodest medico depingere pentagonum quadratum, etiam circulo caveam et chordam arcus invenire?»

99 Vor allem in Padua und Bologna nach dem Vorbild von Pietro d'Abano und Taddeo Alderotti.

100 Vgl. hierzu Goehl (1984) (wie Anm. 87), I, S. 23.

101 Avicenna wurde so auch von Guido im «Liber mitis» am häufigsten zitiert, vgl. Goehl, loc. cit.

102 Avicenna wurde kurze Zeit später auch in Salerno und im folgenden 13. Jahrhundert auch in Padua und an anderen italienischen Universitäten rezipiert.

103 Die von Guido kritisierte Verachtung des Quadriviums (bzw. - aus seiner Sicht - der «artes» überhaupt) wäre natürlich ganz im Sinne Petrarcas gewesen, der den Scholastikern ja gerade ihr Interesse für «nutzlose» naturwissenschaftliche Probleme vorwarf. 


\section{Summary}

\section{Mediaeval criticism of the physician-examples and tendencies from the 6th to the 12th century}

The author presents examples of a critical attitude towards medicine and physicians which may be observed, with changing tendencies, throughout the Middle Ages. First, medicine was despised as a mechanical art or suspected of paganism because of its literary sources. Later, the divine nature of disease and healing on the one hand and the uselessness of medicine on the other were stressed. Finally, the integration, at the universities, of medicine in the system of scholastic learning was criticized by more practically minded men. Similar criticism may be observed in the Islamic world. As valiant defenders of medicine, the author quotes the learned Isidore of Sevilla (d. 636) and the famous Rhazes (b. 865) as well as the anonymous author of the «Lorscher Arzneibuch» of the 8th century.

\section{Résumé}

\section{La critique du médecin au Moyen Age: exemples et tendances du $6^{e}$ au $12^{e}$ siècle}

A l'aide d'exemples, l'auteur présente les principales tendances de la critique faite au médecin pendant le Moyen Age (du $6^{c}$ au 12e siècle). Au début, elle est l'œuvre principalement des idéologues chrétiens (Grégoire de Tours, Bernard de Clairvaux) qui stigmatisent la médecine comme étant une discipline païenne. Avec la fondation des universités, la polémique s'engage dans une dispute avec la scolastique. La question des honoraires de la consultation devient également objet de critiques.

Dr. med. Dr.phil. Klaus Bergdolt

Institut für Geschichte der Medizin

Koellikerstrasse 6/R

D-8700 Würzburg

oder :

Centro tedesco di studi veneziani

Palazzo Barbarigo della Terrazza

San Polo 2765/A

I-30125 Venezia 